\title{
Visual Dysfunction as the First Presentation of Oligodendroglioma - A Case Report
}

\author{
Archana Thool' ${ }^{1}$, Ruta Walavalkar ${ }^{2}$ \\ ${ }^{1,2}$ Department of Ophthalmology, Jawaharlal Nehru Medical College, Datta Meghe Institute of Medical Sciences \\ (Deemed to Be University), Sawangi Meghe, Wardha, Maharashtra, India.
}

\section{INTRODUCTION}

Oligodendrogliomas are one of the most rare and slow-growing brain tumours known to neuroscience. They compromise 0.9 - $4 \%$ of all brain tumours and 3 to 12 $\%$ of all gliomas. ${ }^{1}$ They usually occur in patients who are in their fourth to sixth decades of life. Patients having this particular tumour can be asymptomatic for years owing to its slow-growing nature. Thus, diagnosis at a much-advanced stage sometimes proves to be fatal to the patients. ${ }^{1}$

Intracranial space occupying lesions (ICSOL) such as an oligodendroglioma sometimes manifest as ocular disturbances early in their course of progression. Most common presentation of ICSOL is headache with or without true localizing signs and symptoms. ${ }^{2}$ Therefore they first present to ophthalmologists for their complaints. Visual field testing and fundus examination along with visual acuity carried out in every patient complaining of disturbance of vision when no obvious ocular cause is found is of utmost importance in early diagnosis of brain neoplasms.

\section{PRESENTATION OF CASE}

A 27-year-old male presented to the ophthalmology outpatient department of our hospital with chief complaints of headache on and off for one year and blurring of vision in both eyes for one month. Patient also complained of having vertigo like symptoms for one week. Patient was averagely built. His vitals were stable. He showed no neurological deficit.

On ocular examination, best corrected visual acuity in right eye was $6 / 9$ and that of left eye was 6 / 12. Colour vision in both the eyes by Ishihara Chart was normal. Anterior segment examination was within normal limits. Pupils of both the eyes were normal in size and reaction to light. No relative afferent pupillary defect was detected. Intraocular pressure by applanation tonometry was $12 \mathrm{~mm}$ of $\mathrm{Hg}$ in right eye and 15 $\mathrm{mm}$ of $\mathrm{Hg}$ in left eye. Extraocular muscle movements in both the eyes were free and full in all directions of gaze. Fundus examination (Figure 1) in both eyes by indirect ophthalmoscopy showed the disc margins all over were blurred, obliteration of cup, elevation of disc and peripapillary nerve fibre layer oedema suggestive of papilloedema. Blood vessels and foveal reflex were normal. The patient was referred to neuro-surgery department of our hospital in view of a strong suspicion of an underlying neurological pathology. On doing a magnetic resonance imaging (MRI) brain scan and under expert guidance of the neurosurgeons, the patient was diagnosed to be having a Grade II oligodendroglioma, a pre-malignant low-grade tumour of the occipital lobe.
Corresponding Author:

Dr. Archana Thool,

F-9, Radhikabai Hostel, Post Graduate Girls Hostel, JNMC, DMIMS (Deemed to Be University), Sawangi Meghe, Wardha,

Maharashtra, India.

E-mail: drarchana8030@gmail.com

DOI: $10.14260 /$ jemds/2021/24

How to Cite This Article:

Thool A, Walavalkar R. Visual dysfunction as the first presentation of oligodendroglioma - a case report. I Evolution Med Dent Sci 2021;10(02):114117, DOI: $10.14260 / \mathrm{jemds} / 2021 / 24$

Submission 09-09-2020,

Peer Review 14-11-2020,

Acceptance 20-11-2020,

Published 11-01-2021.

Copyright (C) 2021 Archana Thool et al. This is an open access article distributed under Creative Commons Attribution License [Attribution 4.0 International (CC BY 4.0)] 


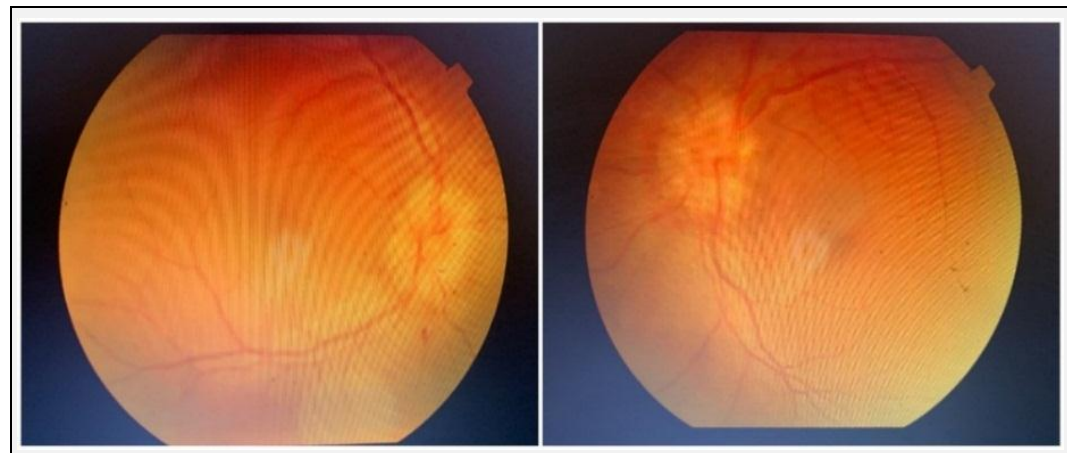

Figure 1. Right Eye \& Left Eye

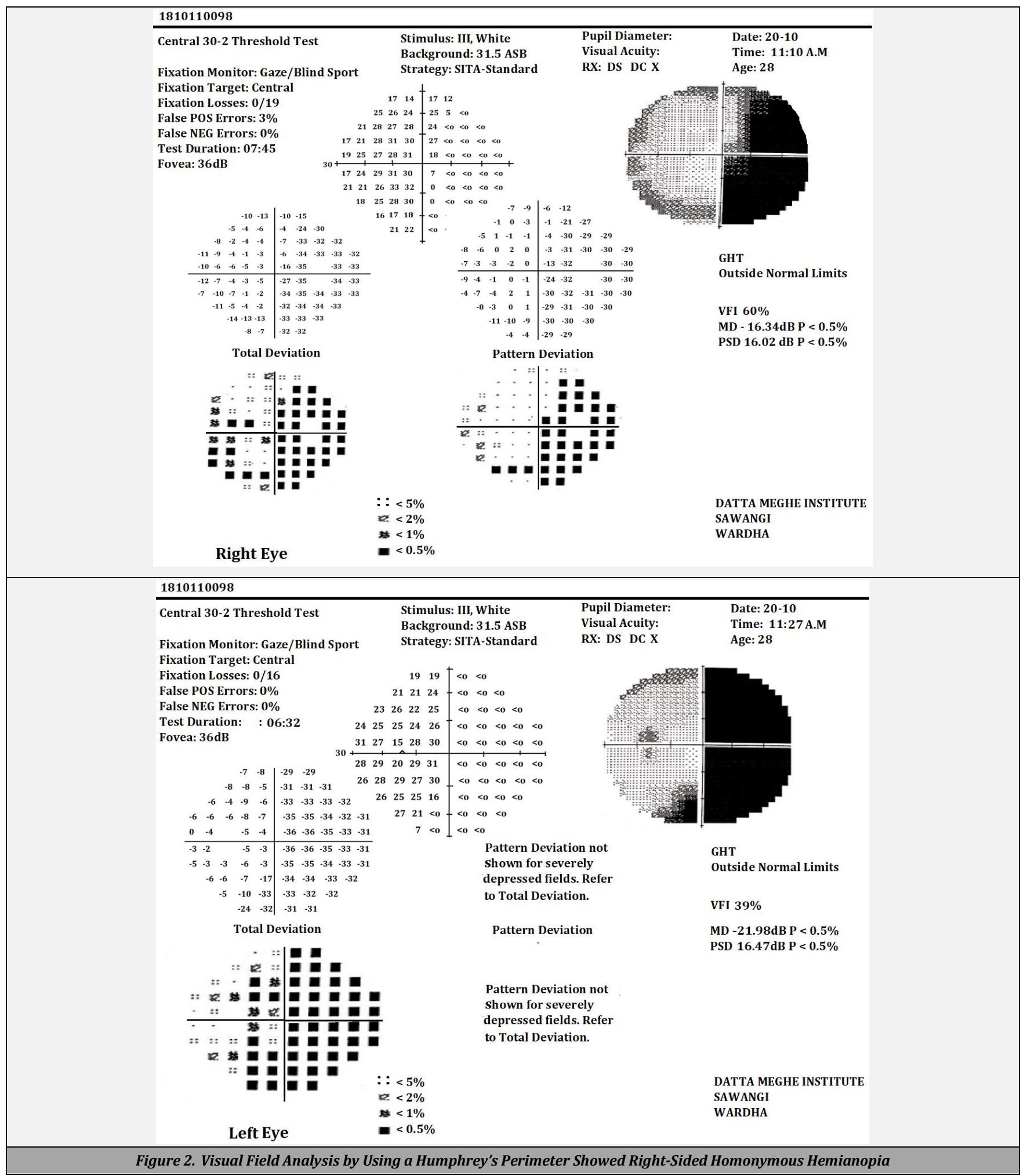


On finding significant visual field defects on perimetry and papilledema on fundus examination, the patient was advised MRI of the brain and orbits to rule out a neuropathology. His MRI of the brain revealed intra-axial ill-defined altered signal intensity mass in the lesion in the left occipital lobe with significant perilesional oedema and mass effect suggesting a possibility of oligodendroglioma.

The patient was referred to department of neurosurgery for further management. The patient underwent craniotomy and excision of the occipital lobe tumour under general anaesthesia.

\section{DISCUSSION}

We have witnessed a revolutionary improvement in the early recognition and diagnosis of ICSOLs in the past century. A commendable contribution by the ophthalmologists in this aspect has been duly noted globally. In the past, almost $75 \%$ to $85 \%$ of all patients with tumours of the brain have papilledema by the time they reached the neurological surgeon as opposed to, in recent times not more than $30 \%$ of the patients who are operated upon for brain tumours have such ophthalmoscopic findings. This is obviously the result of the earlier recognition of these tumours and has enabled the neurosurgeon to improve the prognosis of the patient postoperatively to a significant level. ${ }^{3}$

The vital role of the ophthalmologist in the diagnosis of brain tumours has long been recognized and was aptly emphasized by Cushing when he called the optic chiasm "the crossroads" where the neurologist, the ophthalmologist, and the neurosurgeon meet.4

In our case, the utilization of the similar fact, that the eye works as a camera of the brain was done. The patient had come to the Ophthalmology OPD with very mild and fairly common ocular complaints for which the treatment is usually giving refractive correction. But owing to the multi-dimensional diagnostic approach used, the changes in the fundus and defects in his visual fields was diagnosed to be a rare ocular manifestation of a slow-growing brain tumour. ${ }^{5}$

Due to its mild presentations in the early and curable stage, this particular rare brain tumour is usually diagnosed at a much advanced stage where complete recovery and rehabilitation of the patient is next to impossible. ${ }^{6}$

Although, in this particular case owing to the prompt actions, at a very early stage of the malignancy, the patient was able to undergo a craniotomy and excision of the tumour with early recovery, thus adding not only quantity but also quality to his life. ${ }^{7-9}$

The tumour removed was sent for histopathological examination and the report confirmed it to be an oligodendroglioma Grade II a pre-malignant low-grade tumour (histopathology slide unavailable).

Patient was discharged on analgesic and antacid after adequate post-operative care and advised review in the neurology department after 15 days.

\section{CONCLUSIONS}

Most of the times, an ophthalmologist is the first to detect a neuro-pathology and hence plays a major role in early detection of most of the ICSOLs. ${ }^{10-12}$ Proper evaluation of symptoms and signs and detailed examination and investigation leads to early detection of ICSOL.13-15 Postoperative evaluation and follow-up of cases are equally important. 16,17

Financial or other competing interests: None.

Disclosure forms provided by the authors are available with the full text of this article at jemds.com.

\section{REFERENCES}

[1] Mørk SJ, Lindegaard KF, Halvorsen TB, et al. Oligodendroglioma: incidence and biological behavior in a defined population. J Neurosurg 1985;63(6):881-9.

[2] Raju KV, Khader AA. Ocular manifestations of intracranial space occupying lesions-a clinical study. Kerala Journal of Ophthalmology 2009;21(3):248-52.

[3] Roncone DP. Papilloedema secondary to oligodendroglioma. Clinical and Experimental Optometry 2016;99(6):507-17.

[4] Bucy PC, Keplinger JE. Tumors of the brain stem with special reference to ocular manifestations. AMA Arch Ophthalmol 1959;62(4):541-54.

[5] O'Rourke JF, Schlezinger NS. Evaluation of ocular signs and symptoms in verified brain tumors. J Am Med Assoc 1955;157(9):695-700.

[6] Sood S, Sharma NK, Nada M, et al. Correlation between CT Scan and automated perimetry on supratentorial tumours. Neurol India 2002;50(2):158-61.

[7] Mørk SJ, Halvorsen TB, Lindegaard KF, et al. Oligodendroglioma. Histologic evaluation and prognosis. J Neuropathol Exp Neurol 1986;45(1):65-78.

[8] Paleologos NA. Oligodendroglioma. Curr Treat Options Neurol 2001;3(1):59-66.

[9] Lebrun C, Fontaine D, Bourg V, et al. Treatment of newly diagnosed symptomatic pure low-grade oligodendrogliomas with PCV chemotherapy. Eur J Neurol 2007;14(4):391-8.

[10] Preuss M, Christiansen H, Merkenschlager A, et al. Disseminated oligodendroglial-like leptomeningeal tumors: preliminary diagnostic and therapeutic results for a novel tumor entity. J Neurooncol 2015;124(1):6574.

[11] Helen 00, Oluwole KE, Folasade A, et al. Ophthalmic manifestations in patients with intracranial tumours. African Journal of Neurological Science 2009;28(1):53-9.

[12] Wadud SA, Ahmed S, Choudhury N, et al. Evaluation of ophthalmic manifestations in patients with intracranial tumours. Mymensingh Med J 2014;23(2):268-71.

[13] Pareek A, Aditya K, Sourya A, et al. Correlation between nephropathy and ophthalmic complications in cases of sickle cell anemia: an entangled association. Indian J Med Spec 2019;10(2):72-5.

[14] Prasad M, Daigavane S, Kalode V. Visual outcome after cataract surgery in rural hospital of Wardha district: a prospective study. Journal of Clinical and Diagnostic Research 2020;14(2):NC04-6. 
[15] Daigavane S, Patkar P. To compare the changes in the corneal endothelium post phacoemulsification surgery with balanced salt solution vs. viscoelastic device. Journal of Clinical and Diagnostic Research 2019;13(12):NC01-4.

[16] Patkar P, Sune P. Evaluation of tear film functions preoperatively and postoperatively in cases with pterygium: a case control study. Journal of Clinical and Diagnostic Research 2020;14(1):10-13.

[17] Mukherji R, Sune P. Contrast sensitivity and visual acuity before and after Nd: YAG capsulotomy in patients with posterior capsular opacification: a prospective study. Journal of Clinical and Diagnostic Research 2020;14(3):05-8. 